\title{
Constraints on rotation of B-type stars from the nitrogen abundance
}

\author{
Jakub Ostrowski ${ }^{1, \star}$, Jadwiga Daszyńska-Daszkiewicz ${ }^{1, \star \star}$, and Henryk Cugier ${ }^{1, \star \star \star}$ \\ ${ }^{1}$ Instytut Astronomiczny, Uniwersytet Wrocławski, ul. Kopernika 11, 51-622 Wrocław, Poland
}

\begin{abstract}
.
We study the effects of rotation on surface abundances of $\mathrm{CNO}$ elements in massive stars. Nitrogen enrichment caused by rotationally induced mixing can help to constrain parameters of observed stars, especially their rotational velocities. Here we present theoretical results and apply them to the star HD 163899.
\end{abstract}

\section{Introduction}

Rotation is one of the most important parameters that determine the evolution and ultimate fate of a star. Rotationally induced mixing strongly modifies the internal structure of a star. Besides the difficulty in modelling the effects of rotation, there is also the problem of determining the rotation velocity from observations, and usually only its projected value is known. In the presence of moderateto-fast rotation, the abundance of surface nitrogen should increase during the course of evolution as an effect of rotational mixing processes (e.g., [2]). Abundances of carbon and oxygen are affected as well. Here, we study this effect and use the observed abundance of nitrogen of the star HD 163899 (B2 II) to constrain its rotational velocity.

\section{Results}

Evolutionary models were calculated with the MESA code ([4-6]) assuming a solar metallicity and chemical composition ([1]), initial masses $M \in[16,26] M_{\odot}$ and initial rotation rates $\Omega<0.5 \Omega_{\text {crit }}$, where $\Omega$ and $\Omega_{\text {crit }}$ are the angular rotational velocity and its critical value, respectively. The considered evolution covers stages from the zero-age main sequence (ZAMS) up to the point where $\log T_{\text {eff }} \approx 4.2$ during a supergiant phase. An increase of surface nitrogen abundance with evolution is clearly visible in the left panel of Figure 1 , where we show ratios of mass abundances, $X\left({ }^{14} \mathrm{~N}\right) / X\left({ }^{12} \mathrm{C}\right)$ vs. $X\left({ }^{14} \mathrm{~N}\right) / X\left({ }^{16} \mathrm{O}\right)$, for representative models. In the right panel we show similar diagrams but for models contained in the error box of HD 163899. Theoretical abundances are compared to the values derived from the spectra obtained for the star. Such comparison allows to constrain stellar parameters, especially the rotation rate. We derived an initial rotation rate in range of $0.26-0.30 \Omega_{\text {crit }}$. All details of our analysis are provided in [3].

\footnotetext{
^ostrowski@astro.uni.wroc.pl

$\star \star$ daszynska@astro.uni.wroc.pl

$\star \star \star$ cugier@astro.uni.wroc.pl
} 

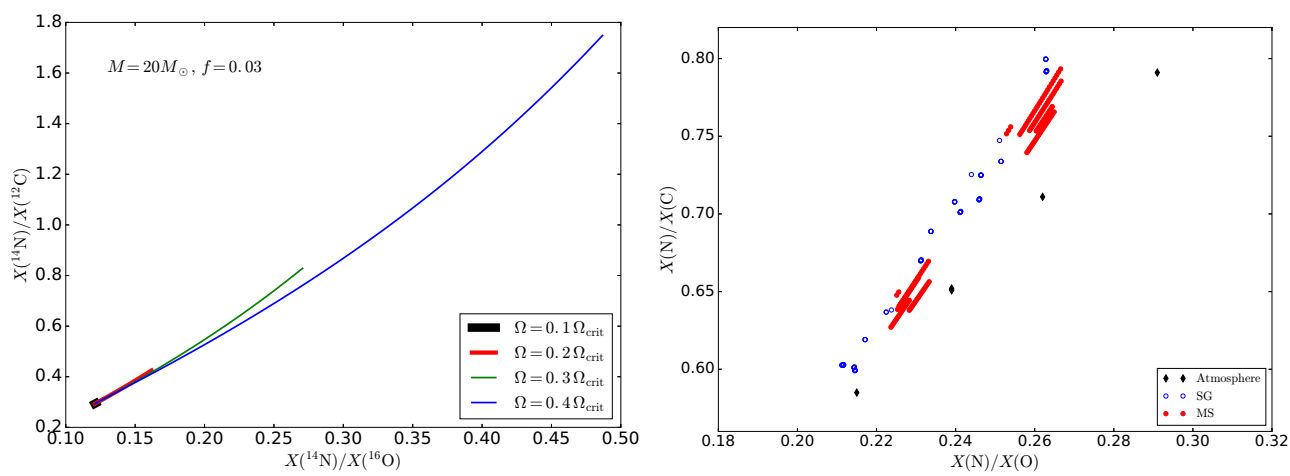

Figure 1. Left panel: the ratios of mass abundances, $X\left({ }^{14} \mathrm{~N}\right) / X\left({ }^{12} \mathrm{C}\right)$ vs. $X\left({ }^{14} \mathrm{~N}\right) / X\left({ }^{16} \mathrm{O}\right)$, for models with initial mass $20 M_{\odot}$, overshooting parameter $f=0.03$ and the four values of the rotation rate at ZAMS. Right panel: the ratios of $X\left({ }^{14} \mathrm{~N}\right) / X\left({ }^{12} \mathrm{C}\right)$ vs. $X\left({ }^{14} \mathrm{~N}\right) / X\left({ }^{16} \mathrm{O}\right)$, showing values derived from atmosphere models with nitrogen enrichment calculated for HD 163899 (black diamonds) and for evolutionary models in the error box of HD 163899 on the main sequence (MS, red dots) and supergiant phase (SG, blue open circles).

\section{Conclusions}

Enrichment of stellar atmospheres in CNO elements due to rotationally induced mixing can be successfully applied to the observed nitrogen-rich stars, as shown for HD 163899. The method helps to obtain the intrinsic rotational velocity disentangled from the inclination angle. More details can be found in [3].

Acknowledgments: Based on the archive "HARPS spectra of CoRoT targets", prepared in the framework of the FP7 project no. 312844 SpaceInn - Exploitation of Space Data for Innovative Helio- and Asteroseismology. This work was financially supported by the Polish NCN grants 2015/17/B/ST9/02082 and 2013/09/N/ST9/00611. Calculations have been carried out using resources provided by Wroclaw Centre for Networking and Supercomputing (http://wcss.pl), grant No. 265.

\section{References}

[1] Asplund, M., Grevesse, N., Sauval, A. J., \& Scott, P., ARA\&A, 47, 481 (2009)

[2] Maeder, A., Przybilla, N., Nieva, M.-F., Georgy, C., Meynet, G., Ekström, S., \& Eggenberger, P., A\&A, 565, A39 (2014)

[3] Ostrowski, J., Daszyńska-Daszkiewicz, J., \& Cugier, H., ApJ, 835, 290 (2017)

[4] Paxton, B., Bildsten, L., Dotter, A., Herwig, F., Lesaffre, P., \& Timmes, F., ApJS, 192, 3 (2011)

[5] Paxton, B., Cantiello, M., Arras, P., et al., ApJS, 208, 4 (2013)

[6] Paxton, B., Marchant, P., Schwab, J., et al., ApJS, 220, 15 (2015) 